\title{
LES DERNIERS LOCUTEURS : AU CROISEMENT DES TYPOLOGIES DES LOCUTEURS DE LANGUES EN DANGER
}

\author{
Jhonnatan Rangel ${ }^{*}$ \\ INALCO, SeDyL (UMR8202 CNRS)
}

\section{Résumé}

Traditionnellement, l'intérêt pour les langues en danger (LED) a été porté sur la description et la documentation des particularités linguistiques de ces langues. Les méthodes et les théories propres de la linguistique descriptive et documentaire ont eu le quasi-monopole sur l'étude des LED jusqu'à récemment, au moment où la figure médiatisée du dernier locuteur a attiré de plus en plus l'attention des chercheurs et du grand public. La réponse des linguistes à cet intérêt croissant a favorisé le développement de typologies de plus en plus complexes de ces locuteurs, prenant en compte des situations très différentes. Dans cette contribution, je discuterai à la lumière de mes travaux sur l'ayapaneco, langue parlée au Mexique, les typologies des locuteurs de LED. Je montrerai que ces caractérisations soulèvent des questions d'ordre théorique et méthodologique complexes qui interrogent des concepts fondamentaux pour la linguistique, telle que la compétence linguistique.

\section{Mots-clés}

locuteurs, ayapaneco, typologie, langue en danger

\begin{abstract}
Traditionally, interest in endangered languages has been primarily focused on the description and documentation of the linguistic particularities of these languages. The methodologies and theories proper to descriptive and documentary linguistics have held the near monopoly in the study of endangered languages until recently, when the mediatized figure of the "last speaker" attracts more and more attention from researchers and the general public alike. The response of linguists to this growing interest has favored the development of increasingly complex typologies of speakers of endangered languages, taking into account the very different situations in which they exist. This article will discuss, through the lens of my work on Ayapaneco, a language spoken in Mexico, the typologies of speakers of endangered languages. I will demonstrate that their characteristics raise complex theoretical and methodological questions that could challenge fundamental concepts for linguistics, such as linguistic competence.
\end{abstract}

\section{Keywords}

speakers, Ayapaneco, typology, endangered language

* L'origine de cette réflexion est une thèse en cours de préparation à l'INALCO (au sein du laboratoire SeDyL du CNRS) et grâce au financement du CONACyT (Mexique). Je tiens à remercier les relecteurs anonymes ainsi que Duna Troiani et Claudine Chamoreau pour leur temps et investissement dans l'amélioration de cet article. 


\section{INTRODUCTION}

Les langues en danger (désormais LED) sans description constituent un matériau original à même de contribuer à affiner des typologies sur des thématiques diverses voire à les questionner. Par ailleurs, la documentation linguistique s'intéresse aussi aux LED car elle cherche à conserver des traces de langues et de cultures qui sont en danger de disparition (Crystal 2000 ; Hale et al. 1992). C'est pourquoi depuis les années 1920, les linguistes sont allés à la rencontre des " derniers locuteurs » de langues menacées de disparition en s'intéressant plus particulièrement aux caractéristiques de ces langues (Costaouec \& Swanton 2015 ; Dixon 1984 ; Evans 1995 ; Harvey 1993 ; Hill \& Hill 1977 ; Miller 1971 ; Palancar 2009 ; Radin 1929 ; Sawyer 1965 ; Zavala 2000). Or, bien que précieux, la plupart de ces travaux n'ont traité la thématique des derniers locuteurs que marginalement. De nos jours, la figure médiatisée du dernier locuteur, en particulier à travers les publications de l'UNESCO ${ }^{1}$, attire de plus en plus l'attention sur les locuteurs de LED. Depuis les années 1970, la réponse des linguistes à cet intérêt croissant a permis le développement des premières typologies de ces locuteurs (Campbell \& Muntzel 1989 ; Dorian 1977 ; Dressler 1981; Sasse 1992). Au début des années 2000, ces études se sont affinées et ont progressivement conduit à l'élaboration de typologies de plus en plus complexes, prenant en compte des situations très différentes (Bert \& Grinevald 2010 ; Evans 2001 ; Hagège 2000 ; Tsunoda 2006).

Dans cette contribution, en m'appuyant sur mes recherches de terrain sur le zoque ayapaneco, je m'attacherai à discuter les typologies proposées pour les locuteurs de LED. Je décrirai, d'une part, la difficulté de caractériser les locuteurs d'une langue parlée au Mexique par moins de 15 personnes et sans transmission intergénérationnelle (Fishman 1991). En effet, ces caractérisations soulèvent des questions d'ordre théorique et méthodologique complexes. D'autre part, j'interrogerai la diversité des profils de ces locuteurs et la façon dont elle est perçue par les locuteurs eux-mêmes.

Cet article s'organise en trois parties. Dans la première partie, je traite les paramètres utilisés traditionnellement pour l'élaboration des classifications des locuteurs de LED. Dans la deuxième partie, je décris précisément les caractéristiques du zoque ayapaneco en tant que LED. Finalement, dans la troisième partie, à partir de la typologie des locuteurs par eux-mêmes, je m'attache à discuter les paramètres présentés dans la première partie dans le but de caractériser les locuteurs du zoque ayapaneco en soulevant quelques questions et en montrant les possibles limites théoriques des typologies présentées. 


\section{Les CLASSIFICATIONS DES LOCUTEURS DE LED}

La réflexion sur les locuteurs des langues en danger a connu un développement substantiel depuis les années 1990, qui s'explique par le fait que la thématique des LED jouit aussi d'un certain intérêt tant auprès des chercheurs que du grand public. Traditionnellement, l'intérêt pour les LED a été porté sur la description et documentation des particularités linguistiques de ces langues. D'une certaine manière, les méthodes et les théories propres de la linguistique descriptive ont pris le quasi-monopole sur l'étude des LED jusqu'à récemment. Dans ce contexte, il est tout à fait normal que les études sur les LED ignorent, la plupart de temps, la thématique des locuteurs au profit de l'analyse de la langue. Or, malgré cette tendance, quelques linguistes ont commencé à s'interroger sur les locuteurs de ces langues.

Nancy Dorian a élaboré, sur la base de critères de compétence linguistique, une première typologie des locuteurs (Dorian 1977), qui a été un outil indispensable pour la conceptualisation des classifications ultérieures élaborées à partir de la diversité des terrains des chercheurs concernés (Campbell \& Muntzel 1989 ; Dressler 1981 ; Hagège 2000 ; Sasse \& Brezinger 1992). De nos jours, les typologies des locuteurs prennent en considération des critères d'analyse multiples car elles ont été enrichies par les réflexions issues de terrains très divers (Tsunoda 2006). À cet égard, la typologie de Bert \& Grinevald (2010) établit des distinctions fines entre les locuteurs de LED en même temps qu'elle synthétise et complexifie les paramètres descriptifs utilisés dans les typologies antérieures. Il s'agit d'un travail tant théorique qu'empirique qui permet de développer une réflexion approfondie sur les profils de locuteurs dans le contexte des LED.

\subsection{Les paramètres de classification}

Au cœur des typologies de locuteurs de LED se trouvent les paramètres descriptifs qui permettent d'établir, a priori, des distinctions précises entre ces locuteurs. Ces distinctions permettent aussi d'identifier des profils typiques qui seraient spécifiques aux situations de LED.

Pour Dorian (1977), le critère de compétence linguistique est central pour identifier les profils des locuteurs. Théoriquement, il serait possible de déterminer le niveau de maitrise de la langue d'un locuteur, ce qui permettrait de distinguer un locuteur donné parmi un continuum de profils possibles. Mais Bert \& Grinevald se méfient du critère de compétence linguistique comme indicateur unique et y ajoutent le niveau d'acquisition de la langue et l'éventuel degré de perte (Bert \& Grinevald 2010, p. 123). Dans leur modèle, ils présentent trois cas de figure : acquisition complète sans perte, acquisition partielle avec possible perte et possible acquisition limitée avec perte importante. La compétence linguistique, l'acquisition et la 
perte seraient donc reliées et leurs interactions permettraient de distinguer plus précisément des profils de locuteurs. En plus du critère linguistique, Bert \& Grinevald considèrent qu'il faut croiser la dynamique de déclin de la langue avec l'âge des locuteurs. Ceci permettrait d'établir une corrélation entre le profil de locuteurs et le niveau de vitalité de la langue. Un dernier facteur à prendre en compte est que dans des situations de LED, il est commun de trouver des individus qui minimisent ou qui surestiment leurs compétences vis-à-vis du linguiste (ibid., p. 124-125).

\subsection{Les profils des locuteurs}

À partir des paramètres mentionnés dans la section précédente, Bert \& Grinevald proposent une typologie de sept profils de locuteurs que l'on trouve dans des situations de LED. Il s'agit de la première typologie des locuteurs de LED en français. Ci-dessous, une synthèse de chaque profil de locuteur est proposée avec son équivalent dans la terminologie anglophone.

Les locuteurs traditionnels sont des individus dont l'acquisition de la langue a été complète et qui ne présentent aucune perte évidente ou 'désacquisition' (Bert \& Grinevald 2010, p. 125). En anglais ce profil est connu comme fluent speaker et Dorian (1981) a proposé de scinder ce profil entre young fluent speakers et old fluent speakers. L'auteur fait ainsi une distinction entre deux générations successives de locuteurs traditionnels qui présentent des variations linguistiques, sans que ces variations soient une source de conflit pour les locuteurs.

Les semi-locuteurs sont des individus dont l'acquisition de la langue a été partielle et qui peuvent présenter des signes d'attrition linguistique. Ces locuteurs possèdent des compétences complètes en réception mais leur compétence en production peut être très variée (Bert \& Grinevald 2010, p. 126). Ce profil de locuteur correspond au semi-speaker dans la typologie de Dorian (1977).

Les sous-locuteurs sont des individus dont l'acquisition de la langue a été très limitée et qui peuvent avoir subi une perte importante des compétences linguistiques. De ce fait, ils possèdent des compétences en réception tandis que leurs compétences en production sont très limitées (Bert \& Grinevald 2010, p. 127). En anglais, ce profil peut recouvrir les dénominations rusty speakers (Sasse \& Brezinger 1992), near passive bilinguals, true passive bilinguals (Dorian 1982, p. 26) et terminal speakers (Dressler 1981 ; Quesada 2000) ; en français, ils peuvent aussi être dénommés sous-usagers (Hagège 2000). Ces dénominations renvoient au même type de locuteurs bien que la définition proposée par chaque auteur puisse être différente.

Les anciens locuteurs sont des individus partiellement compétents, soit en perception / compréhension, soit en production. Ces locuteurs ont été capables, dans 
le passé, de parler la langue mais ils souffrent d'une perte importante dans le présent (Bert \& Grinevald 2010, p. 127). En anglais, on utilise le terme rememberers (Campbell \& Muntzel 1989; Sasse \& Brezinger 1992) pour ce profil de locuteurs.

Les locuteurs fantômes nient leurs connaissances linguistiques bien qu'ils puissent posséder un certain niveau de compétence (Bert \& Grinevald 2010, p. 128). De ce fait, on ignore leur véritable compétence linguistique. En anglais on les appelle disclaimers (Dorian 1986).

Les néo-locuteurs sont des individus qui ont appris la langue dans un contexte de revitalisation linguistique. Leur acquisition de la langue résulte donc d'un effort conscient dans le contexte spécifique de la revitalisation linguistique (Bert \& Grinevald 2010, p. 129). En anglais, les new speakers sont récemment à l'origine d'une production importante de travaux en sociolinguistique (O'Rourke \& Pujolar 2015 ; O’Rourke, Pujolar \& Ramallo, 2014).

Finalement, la dénomination dernier locuteur est utilisée par le grand public, les communautés ou les linguistes. Elle correspond à une étiquette générale qui renvoie plutôt à un statut social ou politique qu'à un type de profil de locuteurs en fonction de critères distinctifs.

\section{LE ZOQUE AYAPANECO : UNE LANGUE EN DANGER}

\subsection{Classification linguistique et distribution géographique}

Le zoque ayapaneco, zoque de Tabasco, Ayapa zoque, zoque d'Ayapa ou ayapaneco est une des langues de la famille mixe-zoque. Les locuteurs appellent leur langue numte oote, numdi oodi ou numde oodi, qui peut être traduit par « vraie voix » ou " parler véritable ».

Les langues de la famille mixe-zoque sont parlées au sud-est du Mexique dans les états de Oaxaca, Chiapas, Tabasco et Veracruz. La famille se compose de six langues associées à la branche zoque et de huit langues regroupées dans la branche mixe pour un total de quatorze langues connues jusqu'à aujourd'hui. L'ayapaneco appartient à la branche zoque et plus particulièrement à la branche du Golfe (Zavala 2015 ; Wichmann 1995).

L'aire géographique dans laquelle cette langue est parlée se limite à la localité d'Ayapa, municipalité de Jalpa de Mendez dans l'État de Tabasco (voir carte 1). Avant l'arrivée des Espagnols dans la région (1518), la communauté de langue ayapaneco se trouvait entourée par des locuteurs de nahuatl (famille uto-aztèque) et de yokot'an (famille maya). Actuellement, la présence de ces groupes s'est réduite considérablement aux proximités d'Ayapa et l'espagnol demeure la langue la plus parlée. 


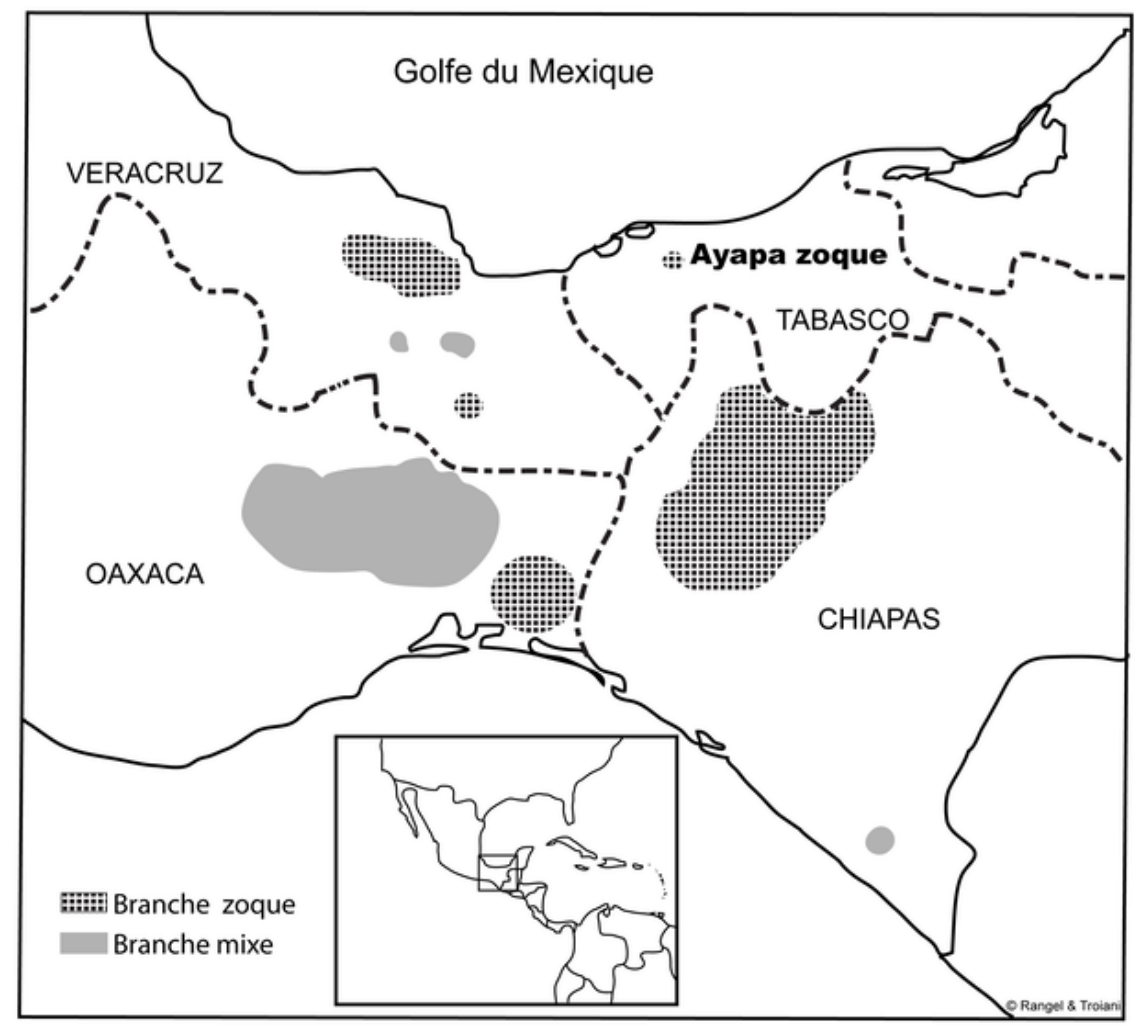

Carte 1. Distribution géographique de la famille mixe-zoque

\subsection{Les recherches sur la langue}

Les recherches publiées sur l'ayapaneco sont rares et fortement lacunaires par rapport aux autres langues de la même famille. La première mention de la langue date de 1960, quand deux chercheurs (Reber \& Webb 1961) du Summer Institute of Linguistics (désormais SIL) ont visité la région et ont recueilli un vocabulaire de 50 mots auprès d'un locuteur qui habitait près d'Ayapa. Cette première liste de mots n'a jamais été publiée. En 1966, l'anthropologue Antonio Garcia de Léon (1971) a recueilli deux textes auprès de deux locuteurs et une petite liste de mots collectée lors de plusieurs courts terrains menés entre 1967 et 1968 dans la région. En 1993, Søren Wichmann a recueilli, auprès de deux locuteurs d'ayapaneco, des données phonologiques dans le but de compléter son travail de reconstruction linguistique de la famille mixe-zoque. Son ouvrage consacre une page à quelques caractéristiques phonologiques de la langue (1995). En 2011, Daniel Suslak a publié un article à propos de l'effet de la médiatisation sur les locuteurs du zoque ayapaneco. 
Il y montre l'existence de variations linguistiques entre deux locuteurs (Suslak 2011). L'auteur a aussi compilé un dictionnaire contenant plus de 6000 items (sous presse).

\subsection{Dynamiques du déclin de la langue}

Afin de présenter une typologie la plus fine possible de locuteurs de LED, il est souhaitable de connaitre et d'inclure les dynamiques du déclin de la langue. À cet égard, depuis les années 1970, la linguistique a commencé à se doter d'outils d'analyse descriptifs dans le but de décrire et de caractériser le plus précisément possible la situation des langues en danger. Pour décrire et évaluer la vitalité du gaélique écossais, Dorian (1977) a utilisé des critères démographiques et linguistiques : nombre et âge des locuteurs, transmission de la langue et compétences linguistiques des locuteurs

Depuis, différentes propositions d'évaluation de vitalité ont été émises, par exemple, l'échelle de transmission intergénérationnelle de Fishman (1991), la grille d'évaluation des experts sur les langues en danger désignés par l'UNESCO (UNESCO ad Hoc Expert Group on Endangered Languages, 2003) et la grille d'évaluation d'Ethnologue liée à la SIL (Lewis \& Simons 2010).

Dans cette contribution, je vais m'attacher à utiliser, comme point d'ancrage théorique, les paramètres d'évaluation des trois grilles citées ci-dessus afin de les appliquer à l'ayapaneco et d'exposer un aperçu général de sa vitalité.

\subsubsection{Le nombre absolu des locuteurs et leur âge}

En 1966, on recensait 150 locuteurs, tous âgés de plus de 35 ans à Ayapa qui, à l'époque, comptabilisait environ 2000 habitants (Garcia de León 1971, p. 213). Ces données mettent en évidence le fait que la transmission traditionnelle de la langue s'était arrêtée plusieurs années auparavant. Par conséquent, on peut affirmer qu'un déplacement linguistique vers l'espagnol était déjà en cours, voire bien installé à la fin des années 60. Dorian (1986) appelle cette période de déplacement linguistique tip, Fishman (1991) le décrit sous le terme language shift et Bert (2010, p. 102) propose le terme de renversement linguistique.

En 2012, j'ai commencé à travailler avec quatre locuteurs ayant entre 60 à 90 ans, lesquels ont identifié dix ou onze personnes de plus pouvant aussi parler la langue. Ainsi, il y aurait environ 15 locuteurs ayant entre 60 à 90 ans. Tous ces locuteurs habitent à Ayapa qui comptabilise aujourd'hui 5500 habitants.

\subsubsection{Les communautés linguistiques de LED}

Dans un cas documenté de LED comme l'ayapaneco, les locuteurs de la langue ne constituent pas une communauté, au sens où les locuteurs ne forment pas un 
groupe dont les membres ont des intérêts communs ou des relations privilégiées. Il existe par contre des sous-réseaux qui s'ignorent car les interactions ne passent pas par la LED (Bert 2010, p. 94).

Pour obtenir le chiffre de 15 locuteurs, j'ai demandé à quatre d'entre eux et à leurs familles de m'indiquer quelles personnes, d'après eux, parlent l'ayapaneco. Les 11 locuteurs désignés semblent correspondre aux sous-réseaux des quatre locuteurs interrogés. On peut alors imaginer que d'autres sous-réseaux de locuteurs peuvent exister dans le village, cachés sous l'anonymat de l'espagnol comme langue de socialisation.

La dynamique de déclin avancé que connait la langue depuis des années contribue à l'atomisation de ces sous-réseaux, ce qui complique la tâche de pouvoir donner des nombres précis de locuteurs. Dans ce contexte, parler de 15 locuteurs d'ayapaneco n'est qu'un chiffre partiel qui reflète la situation du déclin déjà bien engagé de la langue.

\subsubsection{Usage de la langue}

Avant 2012, les locuteurs d'ayapaneco étaient très isolés et ne parlaient la langue que s'ils se rencontraient dans la rue par hasard. On peut donc avancer l'hypothèse qu'entre $1966^{2}$ et 2012 l'utilisation (ainsi que le nombre de locuteurs) aurait diminué progressivement au point que les locuteurs passaient complètement inaperçus dans le village.

À partir de 2012, quatre locuteurs et leurs familles, sous l'impulsion de l'Institut National des Langues Indigènes (désormais INALI), se sont engagés pour la sauvegarde et la transmission de la langue. Ces quatre locuteurs se réunissent deux ou trois fois par semaine pour donner des cours aux enfants du village. Ils ont donc entamé un processus d'enseignement de la langue comme langue seconde auprès d'enfants. C'est lors de ces moments d'enseignement qu'ils parlent la langue entre eux et avec les enfants. En ce qui concerne le reste des locuteurs recensés, on ignore le degré d'usage de la langue dans leur quotidien. Toutefois, vu le nombre restreint de locuteurs, on peut émettre l'hypothèse que l'usage de la langue reste très limité dans le quotidien des locuteurs qui ne sont pas engagés dans l'enseignement de la langue.

\subsubsection{Langue à tradition orale}

Le zoque ayapaneco, tout comme la plupart des langues de la Mésoamérique, est une langue qui n'a pas développé un système d'écriture propre et dont la transmission était orale et réalisée dans le cadre familial. La rupture de cette transmission s'est instaurée autour des années 1940 conjointement au renversement linguistique vers l'espagnol.

2 Année de la première mention dans la littérature du nombre et de l'âge des locuteurs. 
De nos jours, la présence de l'ayapaneco connaît un certain élan, du moins dans les pratiques langagières des quatre locuteurs engagés dans la revitalisation de la langue. De même, pour la première fois, sont publiés des matériaux pédagogiques que les locuteurs fabriquent eux-mêmes en s'inspirant du système d'écriture espagnol dans le but d'enseigner la langue aux enfants du village. Hors du contexte d'enseignement de la langue, la présence de l'ayapaneco dans les pratiques langagières quotidiennes du village est encore inexistante.

\subsubsection{Attitudes et politiques linguistiques}

$\mathrm{Au}$ Mexique, la loi sur les droits linguistiques ${ }^{3}$ garantit depuis 2003 le soutien de toutes les langues du pays. L'ayapaneco serait hypothétiquement dans la même situation que l'ensemble des langues du pays. Or, l'ayapaneco, tout comme la plupart des langues indigènes du pays, n'est pas présente dans l'éducation publique, le commerce, la santé, les médias ou la justice. C'est en 2012 que, pour la première fois, le gouvernement, par le biais de l'INALI, a parrainé des cours d'ayapaneco dans la communauté. Cependant, ces cours ne sont pas reconnus par le système d'éducation publique et sont basés sur le volontariat, tant de ceux qui enseignent que des apprenants (Chamoreau 2014).

$\mathrm{Au}$ vu de la situation actuelle qui a émergé en 2012, on est en mesure de dire que les quatre locuteurs actifs et leurs familles sont motivés pour la sauvegarde et la transmission de la langue. Cette attitude positive récente vis-à-vis de leur langue trouve son origine, en partie, dans le travail de sensibilisation que l'INALI a mené dans le village. Or, les quatre locuteurs engagés pour la sauvegarde et la transmission de la langue et leurs familles sont les moteurs principaux de ce changement récent d'attitude. En ce qui concerne les 11 autres locuteurs, on sait, d'après l'INALI et les locuteurs-mêmes, qu'ils sont trop âgés ou malades ou simplement indifférents pour s'impliquer dans des discussions épilinguistiques.

En ce qui concerne le reste de la population du village, on voit une augmentation significative du nombre d'enfants qui vont régulièrement aux cours d'ayapaneco. En 2012, année du début des cours d'ayapaneco ainsi que lors mes premières observations sur le terrain, 15 enfants assistaient régulièrement aux cours de langue. Au moment où j'écris ces lignes, 60 enfants assistent régulièrement aux cours, ce qui suggère un intérêt croissant pour la langue dans le village ces dernières années.

\subsubsection{Une langue sérieusement en danger}

Une fois qu'on a présenté les faits observables du contexte du déclin de la langue, on peut conclure que la mise en danger de la langue a commencé autour des années 40 lors de la rupture de la transmission dans le village. Elle a pu/dû être suivie

3 Voir Barriga \& Martin (2010) pour un aperçu des politiques linguistiques au Mexique. 
d'une période préalable de bilinguisme espagnol/ayapaneco dans les pratiques langagières de la population. Ce bilinguisme est devenu progressivement un quasi monolinguisme espagnol dans les interactions quotidiennes, jusqu'à ce que la langue ait disparu complètement du cadre familial, entraînant une interruption dans la transmission entre générations.

De nos jours, la langue est certes sérieusement en danger, car le nombre restreint des locuteurs ainsi que leur âge annonce sa disparition imminente dans un futur proche. Néanmoins, la tentative de revitalisation impulsée à l'intérieur de la communauté et soutenue par des institutions de l'État est un fait inédit dans l'histoire de cette langue.

Il est encore tôt pour savoir quelle sera l'issue de ce nouvel élan d'intérêt pour la langue et s'il sera suffisant pour « sauver » l'ayapaneco de la disparition. Pour l'instant, les efforts de revitalisation se concentrent sur l'enseignement de la langue, dans un contexte institutionnalisé, quelques heures par semaine, ce qui est évidemment insuffisant pour inverser le changement de langue (language shift).

\section{LES PROFILS DE LOCUTEURS D’AYAPANECO}

Après avoir présenté les typologies de locuteurs dans la première section et les dynamiques du déclin de la langue dans la deuxième section, la troisième partie de ce travail sera consacrée à la description des caractéristiques des locuteurs d'ayapaneco.

Depuis le début de mon travail de recherche, j'ai eu l'opportunité de travailler avec quatre locuteurs différents. Don Juan a environ 80 ans, Don Mario et Don Cesar environ 70 ans et Don Carlos environ 60 ans $^{4}$. Tous appartiennent donc à la génération des grands-parents. Les quatre locuteurs ont toujours habité à Ayapa où ils sont nés et ont grandi. Ils déclarent avoir appris l'ayapaneco comme langue première, l'espagnol ayant été introduit après, quand ils avaient entre six et sept ans, en particulier dans le contexte scolaire.

\subsection{Le mythe des derniers locuteurs d'ayapaneco}

On a évoqué dans la première section le fait que l'étiquette « dernier locuteur » est plutôt un statut social et, dans certains cas, politique au sein d'une communauté. Dans le cas du zoque ayapaneco, cette étiquette a été aussi utilisée par les médias depuis la première mention de la langue sur internet en 2001. Ils évoquaient la figure des deux derniers locuteurs d'ayapaneco, Don Juan et son frère Don Arturo. Lorsque Don Arturo est décédé il y a quelques années, Don Cesar a, à son tour, été connu comme l'un des deux derniers locuteurs d'ayapaneco.

4 Les noms ont été modifiés et les âges ont été arrondis (à la dizaine) pour préserver l'anonymat des locuteurs et leurs familles. 
Cette médiatisation de la langue à partir du mythe des derniers locuteurs a eu un impact négatif pour les locuteurs et leurs familles. ${ }^{5}$ En effet, les autres locuteurs se sont sentis longtemps dépossédés de leur propre langue, car dans le village on ne les considérait pas comme des locuteurs d'ayapaneco, bien que Don Juan et Don Cesar n'aient jamais contesté l'existence d'autres locuteurs de la langue. Les médias continuent encore de rendre visite à Don Juan, mais aussi à Don Cesar. Chaque mois sur internet, il y a une nouvelle note journalistique qui évoque le mythe des derniers deux locuteurs d'ayapaneco.

Avant cette médiatisation, les locuteurs étaient discriminés à l'école et dans la rue lorsqu'ils pratiquaient leur langue. De nos jours, cette discrimination a été remplacée par l'intérêt que suscite la figure du dernier locuteur. Des journalistes et des visiteurs $^{6}$ internationaux arrivent régulièrement dans le village pour " rencontrer » les derniers locuteurs d'une langue précolombienne proche de l'extinction, comme s'il s'agissait d'espèces en voie de disparition que l'on va voir dans les zoos.

Les locuteurs d'ayapaneco ont souffert d'une attention pernicieuse venant de l'extérieur, à laquelle ils n'étaient pas habitués et qui a modifié les relations entre eux et le reste de la population du village. Ceci aussi contribue au fait que les autres locuteurs se cachent et préfèrent rester dans l'anonymat (locuteur fantômes). On ne peut donc pas conclure que Don Juan et Don Cesar sont réellement les derniers locuteurs d'ayapaneco car d'autres peuvent se dissimuler (de façon consciente ou pas) et se positionner en dehors des sous-réseaux sociaux de Don Juan et Don Cesar, mais aussi de Don Carlos ou de Don Mario.

\subsection{Les locuteurs d'après les paramètres distinctifs linguistiques}

On a vu dans la première section que les typologies de locuteurs incluent parmi les paramètres linguistiques distinctifs la compétence, l'acquisition et la perte de la langue, qui sont des paramètres liés. Et pour la plupart d'entre nous, un locuteur est quelqu'un qui peut parler une langue déterminée.

Une première problématique apparaît lorsqu'on veut déterminer le niveau de maîtrise de la langue d'un locuteur qui parle une langue sérieusement en danger comme l'ayapaneco. Qu'est-ce qu'on entend par « compétence linguistique » quand les interactions entre les locuteurs sont sporadiques et, de ce fait, l'usage de la langue très limité ?

On pourrait être tenté de simplifier la caractérisation en disant qu'un locuteur doit être capable de s'exprimer couramment dans différents contextes où la langue

5 Voir l'article de Suslak (2011) sur l'origine et l'effet de la médiatisation sur les locuteurs de l'ayapaneco.

6 Voir le récit de Daniel Suslak sur la façon dont des acteurs externes à la communauté se sont servi du mythe des derniers locuteurs d'ayapaneco. Disponible sur http://stories.schwa-fire. com/who_save_ayapaneco 
est utilisée même si les interactions entre les locuteurs sont sporadiques. La problématique centrale est donc de pouvoir évaluer la compétence linguistique des locuteurs d'ayapaneco alors que la langue n'est pas utilisée dans la majorité des pratiques langagières en raison des dynamiques de déclin de la langue.

\subsubsection{Outils d'évaluation de la compétence linguistique}

$\mathrm{Si}$, dans les pratiques langagières quotidiennes des locuteurs, la présence de l'ayapaneco est très sporadique, cette absence pourrait être contournée en tentant d'évaluer leur compétence au moyen de tests linguistiques. Pour ce faire, il faudrait concevoir des tests établissant un seuil minimal de compétence pour pouvoir discriminer entre différents profils de locuteurs. Bien que concevable, cette solution est problématique car elle soulève la question de la caractérisation-même du seuil de compétence et des outils pour le définir. Concentrons-nous alors sur la compétence linguistique.

La notion de compétence linguistique a fait couler beaucoup d'encre dans la littérature scientifique depuis les années 60 et le débat théorique est toujours ouvert. Commençons par Chomsky qui propose une nouvelle définition de la compétence, qui doit être distinguée de la performance. La première notion renvoie à une réalité mentale sous-jacente tandis que la seconde renvoie à l'emploi effectif de la langue (Chomsky 1965).

La sociolinguistique variationniste, pour sa part, conçoit les performances discursives comme des échantillons représentatifs qui rendent compte de la nature probabiliste de la compétence (Cedergen \& Sankoff 1972). Tandis que chez Hymes, la notion de compétence (en tant que connaissance sous-jacente) nécessite un point de vue socioculturel pour être opérationnelle. L'auteur s'éloigne ainsi de la conception chomskienne de la performance comme réalisation individuelle du système sous-jacent de la langue et il parle plutôt de compétence de communication et d'événement interactionnel (Hymes 1972).

Actuellement, pour la plupart des linguistes travaillant dans la documentation et description des langues en danger, le choix méthodologique le plus répandu est de recueillir des données à partir d'élicitations linguistiques, de conversations, de narrations et de divers stimuli (Woodbury 2003). De tels outils ont ainsi été utilisés, dans des contextes similaires à celui de l'ayapaneco, pour tenter d'évaluer la compétence linguistique des locuteurs et ainsi sélectionner les informateurs les plus susceptibles de participer à un projet de description ou documentation linguistique. Voir par exemple Bert (2010) avec les locuteurs du franco-provençal et Dorian (1977) avec les locuteurs du gaélique écossais.

Ce choix méthodologique s'appuie sur la typologie des genres linguistiques proposée par Himmelman (1998, 2004), une typologie influencée par la notion 
de compétence communicative de Hymes qu'on vient d'évoquer. La typologie de Himmelman classe les pratiques langagières appelées communicative events (désormais $\mathrm{CE}$ ) dans un continuum qui va des pratiques les plus spontanées (observed communicative events) aux pratiques les moins spontanées (staged communicative events). Dans le cadre de ce travail, nous adoptons cette typologie des genres proposée par Himmelman.

\subsubsection{Limites des outils d'évaluation}

A priori, utiliser la classification des CE nous permettrait d'évaluer la compétence linguistique des locuteurs dans des contextes de déclin comme celui de l'ayapaneco. On trouve des cas documentés qui font état de performances des locuteurs si variées qu'elles ne constituaient pas un continuum linéaire mais multidimensionnel (Bert 2010 ; Elmendorf 1981 ; Guillaume 2010) ; en d'autres termes, certaines situations montrent des locuteurs capables de raconter une histoire mais incapables de soutenir une conversation ou vice versa. On parle alors de compétences variées en fonction des CE.

Ce constat de compétences variées en fonction des CE est aussi présent chez les locuteurs d'ayapaneco. Avec Don Juan, les sessions d'élicitation linguistique, de narrations et de conversations avec les autres locuteurs sont riches en contenu. Mais les sessions avec divers stimuli sont nettement moins productives, à tel point qu'après quelques tentatives, nous les avons arrêtées. Concernant le travail avec Don Carlos, les sessions de narrations et de stimuli sont riches en contenu tandis que les séquences d'élicitation linguistique et de conversations ont produit moins d'interactions et moins de données. Pour finir, les séances d'élicitation linguistique, de stimuli et de conversations avec les deux derniers locuteurs, Don Mario et Don Cesar, ont permis de collecter des données intéressantes sur le plan du contenu et des interactions. Concernant les narrations, elles se sont révélées être une réelle épreuve pour Don Cesar, tandis que l'aisance de Don Mario dans ce domaine est évidente.

Don Juan, Don Carlos, Don Mario et Don Cesar peuvent être caractérisés comme des locuteurs de la langue même si leurs performances dans les CE permettent d'affirmer qu'ils ont des compétences variées. Ils appartiennent à la même génération (grands-parents) et de ce fait, ils peuvent être caractérisés comme des locuteurs traditionnels. Cette caractérisation préliminaire des locuteurs d'ayapaneco peut être affinée en fonction de la diversité de leurs compétences analysées en fonction des CE.

Une deuxième problématique à considérer est qu'au sein d'un même CE, par exemple l'élicitation linguistique, la compétence des locuteurs semble être hétérogène. Ainsi, la compétence linguistique de Don Juan est différente selon qu'il 
s'agit de traduire des phrases vers l'espagnol ou vers l'ayapaneco. En revanche, Don Mario traduit avec aisance vers les deux langues. On perçoit alors la variété des compétences inter et intra $\mathrm{CE}$ en fonction du locuteur, ce qui complique l'évaluation globale des compétences linguistiques et ne permet pas de proposer une classification claire et distincte des locuteurs.

Il est difficile d'expliquer les causes de ces différences car les caractéristiques sociales des quatre locuteurs sont apparemment homogènes. Pour le moment, on peut juste constater que la notion de compétence linguistique est complexe à saisir, du moins à partir de paramètres linguistiques uniquement. Il faut donc aller au-delà et chercher des pistes différentes en croisant tous les paramètres évoqués.

\subsection{Les locuteurs à la lumière des paramètres de l'acquisition et de la perte de compétences}

On a postulé, dans la deuxième partie, l'hypothèse que la rupture de la transmission de l'ayapaneco s'est instaurée autour des années 1940. Les quatre locuteurs sont nés avant ce renversement linguistique, ce qui a priori a favorisé leur acquisition de la langue ayapaneco. Il est cependant complexe de déterminer s'ils ont eu une acquisition complète ou partielle de la langue car il faut définir ce qu'on entend par ces termes dans le contexte ayapaneco. Commençons donc par explorer le niveau d'acquisition.

Si on part du fait que les locuteurs ont acquis l'ayapaneco comme langue première, alors il est tout à fait possible qu'au sein de leur famille la communication ait été uniquement en ayapaneco avant le renversement linguistique. Les quatre locuteurs déclarent n'avoir parlé qu'ayapaneco les premières années de leur vie. Dans ce contexte, l'acquisition de la langue dans leur enfance a été régulière et soutenue avec leurs parents, surtout pour Don Juan qui est le plus âgé des quatre locuteurs. En revanche, l'acquisition de la langue par Don Carlos a probablement été plus limitée car il est plus jeune que Don Juan, et le plus jeune des quatre locuteurs.

On peut alors postuler un continuum en fonction du niveau d'acquisition de la langue : à une extrémité, est positionné Don Juan ; Don Mario et Don Cesar se trouvent dans une position intermédiaire et Don Carlos se situe à l'extrémité opposée à Don Juan. Ce continuum est uniquement indicatif car il repose sur l'hypothèse que l'âge est un indicateur fiable du niveau d'acquisition de la langue. Or, d'autres facteurs peuvent intervenir comme l'exposition à la langue, ou l'usage de la langue dans les communications entre adultes et enfants d'une part, et entre enfants d'autre part. 


\subsection{Niveau d'usage, d'exposition à la langue et compétence linguistique}

Dans sa typologie des locuteurs du francoprovençal et de l'occitan, Bert mentionne que les locuteurs les plus compétents sont ceux qui sont nés avant le renversement linguistique car ils ont eu une acquisition complète de la langue et ont profité d'une exposition continue à celle-ci, qui découle d'un usage quotidien. Ce sont donc des locuteurs natifs considérés comme des locuteurs traditionnels (Bert 2010, p. 104).

On sait que l'usage de l'ayapaneco a diminué progressivement dans le village à partir du renversement linguistique, il y a environ 60 ans. L'exposition à la langue a par la suite progressivement diminué au point qu'elle a été très limitée ou très sporadique pour les locuteurs les plus jeunes comme Don Carlos. On peut donc imaginer une période où l'usage et l'exposition à la langue se seraient dramatiquement réduits, même au sein du foyer.

Cette réduction dans l'usage de la langue aurait contribué à la perte des compétences linguistiques des locuteurs d'ayapaneco. On peut dessiner une échelle croissante en fonction de la perte des compétences par rapport à l'âge des locuteurs. Ainsi, la perte des compétences linguistiques a été moins importante pour Don Juan car il a été exposé plus de temps à la langue, ce qui lui a donné plus d'opportunités pour la parler avec son entourage. La perte des compétences a été plus importante pour Don Mario et Don Cesar car la langue s'utilisait déjà moins. Finalement, à l'extrémité de cette échelle de compétences, se trouve celle de Don Carlos, pour qui la langue ayapaneco était encore plus rarement parlée dans son entourage lorsqu'il était jeune.

Don Carlos et Don Cesar sont nés et ont grandi dans une famille nombreuse qui parlait ayapaneco. Dans ce contexte, on peut imaginer que bien que la dynamique du déclin de la langue fût engagée, la langue continuait à être utilisée au sein de la sphère familiale.

Pour sa part, Don Juan avait un partenaire linguistique : son frère Don Arturo. Les deux frères étaient très proches jusqu'à la mort de Don Arturo il y a une dizaine d'années. Dans cette même famille, le fils de Don Juan pourrait être considéré comme un néo-locuteur car il apprend la langue depuis peu et dans un effort conscient. Il a cependant déjà les compétences linguistiques qui lui permettent de comprendre parfaitement son père bien qu'il lui réponde toujours en espagnol. On peut alors postuler que Don Juan a profité d'une exposition et d'une utilisation ininterrompues de la langue au moins dans la sphère familiale jusqu'à ces dernières années, et ce bien que la langue ait presque disparu de la vie publique.

Don Mario a été longtemps en contact étroit avec des cousins qui parlaient ayapaneco lorsqu'il était jeune. Cela lui aura permis d'être exposé à la langue plus longtemps malgré les dynamiques du déclin de la langue dans le village. En 
revanche, aucune des épouses de Don Mario, Don Carlos, Don Cesar et Don Juan ne déclare parler la langue. J'ai cependant constaté lors de mes sessions de travail avec eux qu'elles semblent comprendre les échanges et par conséquent cacher leur compétence linguistique (locuteurs fantômes).

À partir de 2012, l'usage de la langue revient, quoique de façon sporadique pour Don Juan, Don Carlos, Don Mario et Don Cesar. Les locuteurs ont davantage de situations pour échanger et ainsi retrouver un certain niveau de compétence perdu avec le temps, même si ce contexte d'utilisation est issu d'un effort délibéré, et produit dans le cadre de l'enseignement de la langue.

\subsection{Pratiques langagières, compétence linguistique et appartenance}

Dans certaines situations de LED, il peut ne pas y avoir de corrélation directe entre compétence linguistique et pratiques langagières (Bert 2010, p. 104). En d'autres termes, un locuteur dont les compétences linguistiques sont observables, au moins à partir d'une perspective externe comme celle d'un linguiste, peut ne pas utiliser la langue dans son quotidien même s'il y a d'autres locuteurs autour de lui. Il s'agit d'un choix conscient de ne pas parler la langue pour des raisons externes à la compétence linguistique.

Evans (2001) décrit une autre situation rencontrée lors de son travail de recherche en Australie. Il propose ainsi le terme de language-owner (désormais LO) pour des individus qui pensent avoir le droit d'être locuteur d'une langue sans que cela corresponde à des compétences réelles. En opposition, le terme de language-users (désormais LU) renvoie à des individus qui utilisent la langue, mais qui ne se perçoivent pas comme des locuteurs à part entière (Evans 2001, p. 250251). Dans la typologie de Bert \& Grinevald, les locuteurs fantômes pourraient se situer du côté des LU. Dans tous ces cas, il s'agit d'un critère d'appartenance à la communauté des locuteurs et non de compétence linguistique.

En ayapaneco, Don Juan, Don Carlos, Don Mario y Don Cesar seraient des LO car ils se perçoivent eux-mêmes comme des locuteurs de la langue. Cependant, ils présentent des compétences linguistiques variées en fonction des $\mathrm{CE}$ et de ce fait, ils sont aussi des LU. Or, le contexte restreint des CE ne permet pas d'attester la présence de l'ayapaneco dans les pratiques langagières quotidiennes des locuteurs, ce qui complique la tâche pour différencier nettement un LO d'un LU dans ce cas spécifique.

En dehors des quatre locuteurs, on ignore s'il y a d'autres personnes à Ayapa qui auraient des compétences linguistiques en ayapaneco, mais qui ne les assumeraient pas (LU/locuteurs fantômes). Une première piste à explorer est la famille proche des locuteurs. Mes observations de terrain m'ont permis de constater que 
les épouses des quatre locuteurs ainsi que le fils de Don Juan ont une compétence linguistique en ayapaneco. Or, cette compétence linguistique est difficilement évaluable car ils la nient catégoriquement depuis le début de mes observations : ils ne se considèrent pas comme des locuteurs.

\subsection{Compétence linguistique et partenaires linguistiques}

Les compétences variées des locuteurs peuvent s'expliquer par des facteurs externes à la langue ainsi que par les tests de compétence utilisés (artéfacts d'évaluation). De même, la présence d'autres personnes (partenaires linguistiques) a une influence directe sur la compétence des locuteurs.

L'influence des partenaires linguistiques dans la compétence linguistique des locuteurs a déjà été documentée dans des contextes comme celui de l'ayapaneco. Il s'agit de l'augmentation ou de la diminution des compétences des locuteurs au contact d'un partenaire linguistique. Evans (2001, p. 273-274) propose le terme amplifier pour ces personnes pouvant avoir un effet d'augmentation de la compétence d'un locuteur. Il documente aussi le cas contraire, autrement dit, des locuteurs qui vont « diminuer» leur compétence au contact de certaines personnes.

Quelles sont les dynamiques existantes entre les quatre locuteurs d'ayapaneco ? Peut-on vraiment parler d'augmentation ou de diminution des compétences linguistiques en fonction du partenaire linguistique ? Des dynamiques observées entre les quatre locuteurs, je peux dégager certains constats. En général, les échanges entre Don Mario et Don César sont nourris. Lorsqu'ils sont ensemble, j'observe que la production narrative de Don Mario coule avec plus d'aisance qu'avec les autres locuteurs. Pour Don Cesar, les séances d'élicitation par stimuli visuel semblent se dérouler avec plus d'aisance lorsque Don Mario interagit directement avec lui.

On peut aussi se demander si la présence du chercheur a un impact direct sur les compétences linguistiques des locuteurs. À cet égard, j'ai observé que lorsque Don Carlos est seul avec moi, il semble être plus à l'aise lors des séances d'élicitation, de narration et de stimuli. Je suis en mesure d'affirmer cela car lors des mêmes CE mais au contact des autres locuteurs, particulièrement de Don Juan, Don Carlos a tendance à se taire au point qu'ils interagissent difficilement ensemble.

Dans cette description des faits observables, je n'ai à aucun moment suggéré que la présence d'un partenaire linguistique a un impact direct sur les formes linguistiques produites par les locuteurs ou que l'interaction d'un partenaire donné génère des «fautes » ou des propositions agrammaticales chez un autre locuteur.

Étant donné que définir la notion de compétence linguistique dans un cas comme celui de l'ayapaneco est assez problématique, je préfère ne pas utiliser les 
termes qualitatifs de diminution ou d'augmentation de la compétence, en revanche je rapporte les différences observables dans les dynamiques d'interaction entre locuteurs, et entre le linguiste et les locuteurs. Lorsque ces dynamiques entre partenaires linguistiques sont favorables, on constate que les pratiques langagières des locuteurs sont plus riches et nourries au sein d'un CE. Par contre, lorsque ces dynamiques sont moins favorables, les pratiques semblent plus pauvres.

\subsection{Croisement de paramètres}

On a montré au début de cette troisième partie que Don Juan, Don Mario et Don Cesar sont des locuteurs qui appartiennent à la même génération et que, de ce fait, ils peuvent être considérés comme des locuteurs traditionnels. De même, ce sont des LO car ils se perçoivent comme des locuteurs d'ayapaneco. En revanche, Don Carlos a souffert d'une acquisition moins complète et d'une perte plus importante par rapport aux autres locuteurs. On ne peut cependant pas le caractériser comme un semi-locuteur car sa compétence linguistique est aussi variée que celle des autres locuteurs, mais dans des contextes différents et en fonction aussi du partenaire linguistique.

Les variations linguistiques entre les locuteurs ne nous permettent pas non plus de distinguer entre un locuteur traditionnel âgé et un plus jeune. En effet, les variations constatées entre les quatre locuteurs les répartiraient différemment, par exemple Don Juan et Don Mario appartiendraient au même groupe tandis que Don Cesar et Don Carlos constitueraient un autre groupe ${ }^{7}$.

\subsection{Classification des locuteurs par les locuteurs}

Jusque-là, on a classé les locuteurs à partir des critères comme la compétence linguistique. Or, dans toute cette discussion nous n'avons pas pris en considération ce que les locuteurs pensent de leurs propres compétences linguistiques. Ceci est pertinent car les catégorisations des linguistes ne correspondent pas toujours à celles des locuteurs (Evans 2001, p. 260).

Don Juan, Don Mario, Don Carlos et Don Cesar (désormais DJ, DM, DC et DCE) s'acceptent comme des locuteurs d'ayapaneco entre eux sans spécifier de différences. Or, au cours de mon travail de terrain, j'ai repéré des périphrases sur les compétences linguistiques qui ont émergé de leur discours à plusieurs reprises, périphrases que j'ai explorées dans nos conversations. Il en ressort une première proposition de typologie des classifications des locuteurs par les locuteurs euxmêmes. Ci-dessous, je présente cette typologie en analysant et illustrant chacun des 4 types.

7 Pour un traitement plus approfondi des variations linguistiques voir Rangel (thèse en cours d'élaboration à l'INALCO). 


\subsubsection{Type 1. ni yodo numdi oodi}

Le premier type correspond à une personne qui ni yodo numdi oodi (parle ayapaneco). Cette catégorie inclut tous ceux qui s'adressent à une autre personne en ayapaneco et qui répondent à une salutation. Cette première définition part d'un événement interactionnel au sein d'un CE spécifique (échange de salutations). En d'autres termes, un locuteur A est au moins capable d'échanger quelques formules de salutation avec un locuteur $\mathrm{B}$ dans la sphère publique.

1) $\mathrm{C}^{8}$ : Comment savez-vous que quelqu'un parle ayapaneco ?

DJ : Car quand on est dans la rue il te parle. ${ }^{9}$

$\mathrm{C}$ : Alors, si je sors dans la rue et lui dis jumna (ça va)?

DJ : Et il répond (A1_17082016EE).

À la notion d'interaction dans la sphère publique s'ajoute la notion d'appartenance à un lieu spécifique, c'est-à-dire Ayapa.

2) C : Comment savez-vous que quelqu'un parle ayapaneco ?

DM : Parce que nous sommes du même endroit et avant, nous nous retrouvions dans la rue où nous savions qu'il parlait, c'est comme ça que nous savons que cette personne le parle (ayapaneco).

DCE : Moi, parce qu'ils me parlent (A34_19082016EE).

Une troisième composante de cette définition est que ces individus étaient déjà identifiés par eux comme des locuteurs avant le renversement linguistique comme on peut le voir dans l'extrait de conversation ci-dessous.

3) $\mathrm{C}$ : Et alors, vous vous rendez compte (que quelqu'un parle la langue) seulement parce que quelqu'un vous parle dans la rue?

DM : Parce que depuis que nous avons commencé à marcher dans ce village, nous savons qui sont ceux qui parlaient (l'ayapaneco) en ce temps-là, quand nous grandissions (A34_19082016EE).

On identifie alors dans cette définition trois paramètres distinctifs d'après les locuteurs : la salutation, l'appartenance au village et l'identification de ces personnes comme des locuteurs avant le renversement linguistique. En d'autres termes, il s'agit d'une définition de locuteur à partir de critères sociaux comme la reconnaissance ou l'identification et non à partir de la compétence linguistique.

D'après les locuteurs, ce profil de locuteur recouvre aussi ceux qui jyo? numdi oodi (connaissent l'ayapaneco). De ce fait, quelqu'un qui a des connaissances de la langue doit être capable de la parler, et inversement le fait de parler la langue sous-entend la connaissance de celle-là.

$8 \mathrm{C}=$ chercheur

9 En gras les mises en relief de l'auteur. 
Les 15 locuteurs recensés entrent dans ce type car ils ont parlé dans le passé, ils parlent aujourd'hui ou ils ont des connaissances de la langue et, de ce fait, ils sont capables de parler la langue s'ils le veulent. Le type 1 se caractérise alors comme une catégorie vaste.

\subsubsection{Type 2. taani yodo gwüü numdi oodi}

Il s'agit d'individus qui dans le passé étaient des ni yodo numdi oodi (parle ayapaneco) mais qui ont perdu des compétences linguistiques à cause du manque de pratique comme on peut le voir dans l'extrait de conversation ci-dessous. Il y a donc un lien entre la perte des compétences et l'absence d'utilisation de la langue.

1) $\mathrm{C}$ : Est-il possible que quelqu'un qui comprend (l'ayapaneco), ce n'est pas qu'il ne le veuille pas, mais qu'il ne puisse pas parler ? Ou pensez-vous que si on a des connaissances, je veux dire, on ne parle pas car on ne veut pas?

DM : Comme je t'ai déjà dit, la langue, si on ne la pratique pas, on ne l'utilise pas, elle ne sort pas bien même si tu veux la parler (A34_19082016EE).

Dans les paroles de Don Juan, «ces individus ne parlent pas bien, ils parlent à l'envers, il y a des mots qui ne sortent pas bien » (A1_17082016EE). Il y a donc des conséquences structurelles qui sont repérables par les locuteurs.

Ce type de locuteur sí ni yodo pero taani yodo gwüü (parle mais il ne parle pas bien) et il ne parle bien car taani pyut gwüü (les mots ne sortent pas bien). En d'autres termes, il commet des erreurs et par conséquent il ne parle pas la langue avec fluidité.

Il semble qu'une partie des 15 locuteurs d'ayapaneco recensés entrent aussi dans ce type. Autrement dit, certaines personnes considérées comme appartenant au type 1 peuvent être inclus dans le type 2 car elles commettent des erreurs (repérables par les autres locuteurs).

Ici, on voit que les deux premiers types peuvent se chevaucher car le type 1 est un macro-type qui englobe d'autres sous-types comme le type 2. Le fait de ne pas bien parler (type 2) n'exclut pas de considérer quelqu'un d'être comme un locuteur (type1) pourvu qu'il réponde aux critères du type 1.

\subsubsection{Type 3. ni mbyadaj pero taani yodo}

À ce type appartiennent toutes ces personnes qui ni mbyadaj pero taani yodo (comprennent mais ils ne parlent pas) lorsqu'on leur parle en ayapaneco. Les locuteurs sont sûrs que ces personnes sont capables de tout comprendre comme on peut le voir dans l'extrait de conversation ci-dessous.

1) C : Y-a-t-il des gens par exemple, pour qui il est possible de connaître ou comprendre (la langue) sans la parler?

DM : Il ne pratique pas, alors ne l'utilise pas.

$\mathrm{C}$ : Par exemple tu lui dis tout et lui, il comprend tout mais il ne répond pas ? 
DM : Il ne répond pas mais il comprend tout ce que tu lui dis (A34_19082016EE).

D'après les locuteurs, ces personnes ni mbyadaj pero taa xyu yodo (comprennent mais ne veulent pas parler). Alors, il s'agit d'un choix délibéré de ne pas parler la langue malgré le fait que ces personnes sont capables de comprendre. Ce choix délibéré d'après les locuteurs est motivé par le fait que, comme ces individus ne parlent pas bien la langue, ils préfèrent s'abstenir de parler comme le montre l'extrait de conversation ci-dessous.

2) DM : ...comme je te dis, ils comprennent mais ils ne parlent pas car ils ne veulent pas, ils ne se décident pas, car parfois la langue ne sort pas bien et à cause de cela ils ne veulent pas parler (A34_19082016EE).

Ces individus alors jyo? pero taani yodo (ont des connaissances mais ne parlent pas la langue) semblent délibérément choisir de ne pas parler en ayapaneco comme on le voit dans l'extrait ci-dessous.

3) $\mathrm{C}$ : Y-a-t-il des gens qui ont des connaissances (de la langue) et avec qui vous avez déjà parlé ?

\section{DJ : Ils ne veulent pas parler \\ C : Ils parlent bien ?}

DJ : Parfois oui mais ils ne veulent pas parler. Ils arrivent chez eux et ils disent « je ne sais rien », pourtant ils parlent. Ce sont des individus renfermés (A1_17082016EE).

D'après les locuteurs, leurs épouses ainsi qu'une partie des 15 personnes recensées appartiennent à ce type. En d'autres termes, des personnes appartenant au type 1 peuvent appartenir aussi au type 3 lorsqu'elles décident de ne pas parler. Le type 1 alors englobe aussi le type 3, car ils ne s'excluent pas entre eux. Dans ce contexte, seuls les paramètres sociaux (vivre à Ayapa et être reconnu comme un locuteur avant le renversement linguistique) sont pris en compte car ces locuteurs, le plus souvent ne saluent pas en ayapaneco, soit parce qu'ils sont âgés, soit parce qu'ils préfèrent saluer en espagnol.

Un même locuteur peut progressivement modifier son comportement et, en conséquence, cela modifiera son appartenance à un type. Par exemple, quelqu'un de type 2 passera au type 3 s'il décide de ne pas parler. Le cas contraire est aussi possible, que quelqu'un identifié comme appartenant au type 3 passe au type 2 lorsqu'il décide de se remettre à parler même s'il ne parle pas bien la langue. À la frontière entre les types 2 et 3 se trouve alors une modification progressive selon que les locuteurs s'expriment ou ne veulent plus s'exprimer en ayapaneco. Il s'agit d'un changement qui s'inscrit progressivement dans le temps. 


\subsubsection{Type 4. ni ye?e yuj numdi oodi}

Finalement, les individus qui ni ye?e yuj numdi oodi (viennent apprendre l'ayapaneco) recouvre l'ensemble des personnes qui comme moi, dans un effort conscient et récent, apprenons la langue. Ainsi, un linguiste, comme les étudiants qui assistent aux cours, peuvent être classés dans ce type.

Par exemple, lorsque j'ai commencé à travailler avec les quatre locuteurs, j'étais catalogué dans le type 4 . Au fur et à mesure que j'ai mené mon travail de recherche dans le village, j'ai constaté que d'autres dénominations m'étaient attribuées. Par exemple, je suis catalogué comme quelqu'un qui appartient au type 4 ni ye? e yuj numdi oodi (vient apprendre ayapaneco) mais aussi plus récemment comme quelqu'un qui appartient au type 1 ni yodo numdi oodi (parle ayapaneco). Or, je ne réponds pas aux critères sociaux (appartenance au village et identification comme locuteur avant le renversement linguistique) mais je réponds au critère de salutation. Or, j'ignore encore si le changement de type qu'ils m'ont attribué est dû uniquement à mes compétences linguistiques (capacité de saluer) ou à d'autres facteurs tels que ma présence constante dans le village ou mon effort conscient d'étudier et d'apprendre la langue. Le type 4 est une situation nouvelle pour les locuteurs et, de ce fait, c'est encore un type ouvert et en pleine définition. Il faudra suivre l'évolution de ce type dans de futures recherches.

\subsubsection{Chevauchement et changements de types}

La figure 1 synthétise le positionnement des quatre types de locuteurs analysés ainsi que les zones de chevauchement temporelles. Les types 2 (désormais T2) et 3 (désormais T3) sont contenus dans le type 1 (désormais T1). Il peut y avoir un chevauchement (en gris) entre le T2 et le T3 lorsqu'une personne est dans le processus dynamique de modifier son comportement (décide de parler ou de ne pas parler). T4 est un type nouveau qui a surgi depuis peu de temps mais il semble que certains locuteurs identifiés comme T4 puissent aussi être identifiés à l'intérieur du T1. Il est encore tôt pour déterminer si dans un futur proche un apprenant d'ayapaneco (T4) pourra passer au T2 s'il ne parle pas bien d'après les locuteurs ou au T3 s'il décide de ne pas parler une fois qu'il a acquis des compétences linguistiques.

Les quatre types de locuteurs analysés permettent de constater qu'il peut y avoir des chevauchements et changements dans le temps entre types. Ainsi, une personne appartenant au T1 1 ni yodo numdi oodi (parle ayapaneco) peut rester dans ce type tout au long de sa vie, comme les 4 locuteurs principaux avec lesquels je travaille, mais d'autres peuvent modifier leurs comportements et changer de type, se retrouvant ainsi dans le T2 taani yodo gwü̈̈ numdi oodi (ne parle pas bien ayapaneco). Ce changement est progressif et visible lorsque les autres locuteurs identifient ce qu'ils considèrent des « anomalies » linguistiques. Ceci 
pourrait s'expliquer par le fait que la caractérisation du T1 ne contient pas de critères explicites sur la compétence autre que d'être capable de saluer. Donc, une personne peut saluer, être du village et ancienne (donc appartenir au T1) mais si lorsqu'elle parle au-delà du salut, elle ne parle pas bien, et elle sera alors classée dans T2. Dans ce contexte, T2 est un groupe particulier du T1 qui est assez vaste.

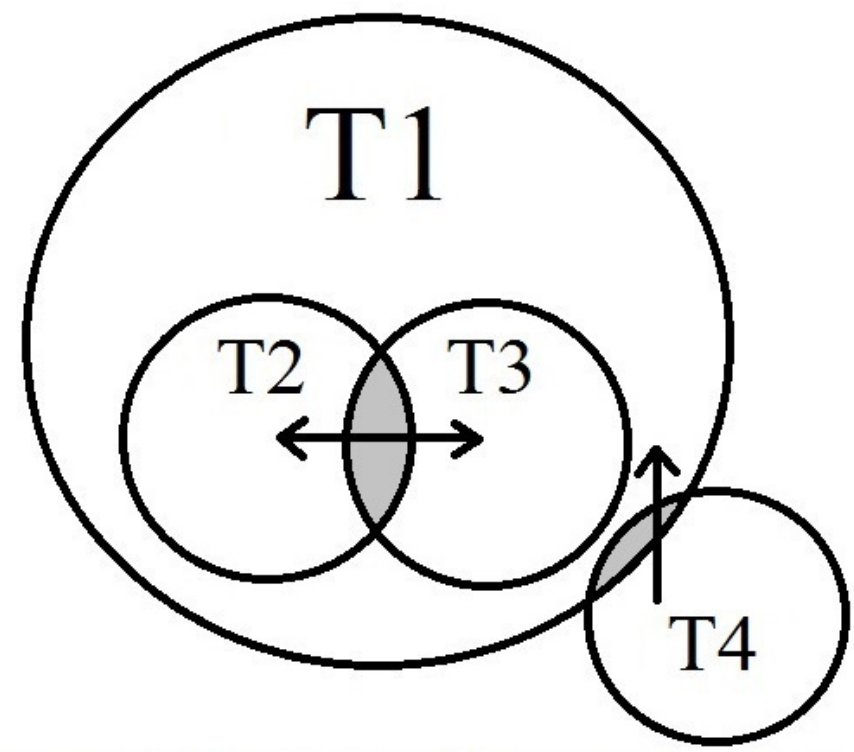

Figure 1. Types de locuteurs: chevauchements et changements de type

Un individu appartenant au T2 taani yodo gwüü numdi oodi (ne parle pas bien ayapaneco) peut changer au T3 ni mbyadaj pero taani yodo (comprend mais ne veut pas parler) s'il décide de ne pas parler en ayapaneco. Il s'agit d'un changement du T2 au T3 motivé par le refus de parler la langue. Or, l'origine de ce refus de parler peut être le fait de ne pas bien parler la langue (T2) comme DM l'a signalé dans l'extrait 5 . Le cas contraire est aussi possible lorsque quelqu'un appartenant au $\mathrm{T} 3$ décide de se remettre à parler la langue, et de ce fait, il passe au T2. On voit donc que la modification du comportement des locuteurs déclenche un changement d'appartenance à un type et ponctuellement il y a un chevauchement entre les T2 et T3. Dans ce contexte, T3 est aussi un groupe particulier du T1.

\section{Conclusion}

Dans cette contribution, je me suis attaché à analyser les typologies des locuteurs de LED, celles proposées par des linguistes mais aussi celle des locuteurs 
d'ayapaneco. Le croisement de ces typologies nous permet de faire quelques remarques générales en guise de conclusion.

T1 partage des caractéristiques avec la définition des locuteurs traditionnels (désormais LT). Or, les LT sont des locuteurs qui ne présentent aucune perte et dans le cas des T1, la perte des compétences linguistiques entraine une sous-catégorisation (T2 et T3) au sein de T1. T2 partage des caractéristiques avec la définition de semi-locuteurs (désormais SML), de sous-locuteurs (SL) ainsi que d'anciens locuteurs (désormais AL). En effet, les T2 sont des individus qui ont perdu des compétences linguistiques bien qu'ils soient identifiés comme T1. Ceci pose un problème conceptuel car SML, SL et AL sont des catégories bien différenciées. De même, T2 est une sous-catégorie de T1 pour les locuteurs d'ayapaneco. En revanche, la sous-catégorisation de SML, SL et AL au sein de LT n'est pas envisageable dans les typologies des linguistes.

T3 partage des caractéristiques avec la définition de locuteurs fantômes (désormais LF) sauf que, pour les locuteurs d'ayapaneco, T3 est aussi identifié comme un sous-type de T1. La relation entre un LT et un LF ne permet pas ce type de chevauchement. Finalement, T4 partage des caractéristiques avec la définition de néo-locuteurs (désormais $\mathrm{NL}$ ) bien que pour les locuteurs d'ayapaneco, une personne de T4 puisse changer et intégrer T1 (comme l'illustre mon cas). Or, dans la typologie des linguistes il n'est pas envisageable qu'un NL devienne un LT.

La figure 2 synthétise le croisement entre les typologies des linguistes et celles des locuteurs d'ayapaneco. Sont donnés entre parenthèses les équivalents dans la typologie des linguistes, les flèches représentent la direction des changements entre types et le grisé, les chevauchements dans le temps.

Cette comparaison met l'accent sur les différences entre les deux approches. Les profils dans les typologies des linguistes, bien que dynamiques, s'excluent entre eux. Un LT ne peut être en même temps un SML, un SL, un AL ou un LF. Mais dans la typologie ayapaneco, le T2 et le T3 sont aussi des T1 car ce type représente un macro-type. Plus remarquable encore, un NL ne peut être un LT car la définition-même ne permettrait pas un chevauchement entre ces deux types. Dans le cas de la typologie ayapaneco, il y un chevauchement dans le temps (passage d'un type à l'autre) du T4, qui est en train de se positionner à l'intérieur $\mathrm{du}$ T1. Ceci illustre le fait que les frontières dans la typologie des locuteurs sont beaucoup plus fluides et dynamiques que dans celle proposée par les linguistes, constituée de catégories imperméables. 


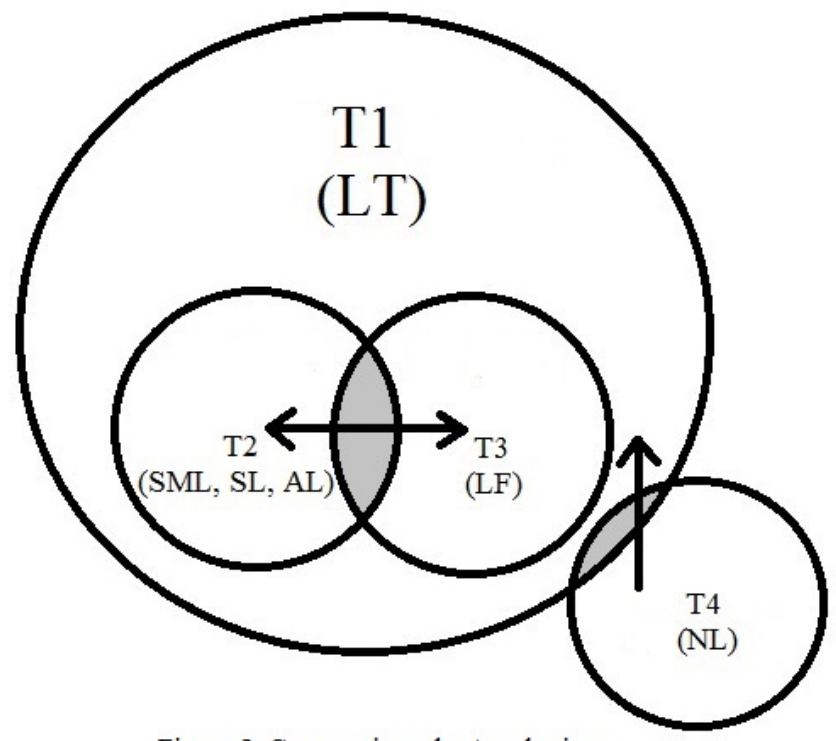

Figure 2. Comparaison des typologies

Cette comparaison des typologies des locuteurs nous permet, d'une part, de repérer les écarts entre nos définitions (de linguistes) et celles des locuteurs. Elle nous permet, d'autre part, d'appréhender et, de ce fait, d'intégrer le dynamisme de ces définitions à nos recherches sur le terrain dans le but de produire des analyses plus détaillées. À cet égard, la typologie des locuteurs par les locuteurs eux-mêmes met en lumière que les frontières entre catégories sont dynamiques et très perméables. En effet, les profils des locuteurs d'ayapaneco sont en constante évolution car les paramètres distinctifs sont redéfinis continuellement. En outre, l'instabilité de ces paramètres est accentuée par les dynamiques du déclin avancé que la langue vit depuis des années.

Le croisement des typologies nous permet encore, grâce à une intégration des définitions propres aux locuteurs, d'enrichir des questionnements d'ordre théorique comme la compétence linguistique ou la définition de locuteurs. En effet, la notion de compétence linguistique, centrale dans les typologies des linguistes, est encore problématique car elle ne nous permet pas de faire une distinction nette entre différents profils de locuteurs d'ayapaneco. De ce fait, on gagne à remettre en cause, avec un nouveau regard, des questionnements d'ordre méthodologique comme l'évaluation de la compétence linguistique ainsi que la classification des locuteurs de LED. 


\section{BIBLIOGRAPHIE}

Barriga Villanueva, Rebeca, \& Martin Butragueño, Pedro, 2010. Historia sociolingüística de México, México, El Colegio de México, Vol. 2.

Bert, Michel, 2010. «Qui parle une langue en danger ? Locuteurs du francoprovençal et de l'occitan en Rhône-Alpes ", Faits de Langues 35/36, 79-115.

Bert, Michel, \& Grinevald, Colette, 2010. «Proposition de typologie des locuteurs de LED, France », Faits de Langues 35/36, 117-132.

Campbell, Lyle, \& Muntzel, Martha Claire, 1989. «The structural consequences of language death », Campbell, Lyle (éd.), Investigating obsolescence, Cambridge University Press.

Cedergren, Robert J. \& Sankoff, David, 1972. « Variable rules: performance as statistical reflection of competence ", Language 50 (2), 333-350.

Chamoreau, Claudine, 2014. "Diversidad lingüística en México: un acercamiento », Amerindia, 37 (1), 3-20.

Chomsky, Noam, 1965. Aspects of the theory of syntax, Cambridge Massachusetts, MIT Press.

Costaouec, Denis, \& Swanton, Michael, 2015. « Classification nominale en Ixcatèque », La linguistique 51 (2), 201-238.

Crystal, David, 2000. Language death, Cambridge, Cambridge University Press.

Dixon, Robert Malcom Ward, 1984. Searching for Aboriginal Languages: Memoirs of a Field Worker, Cambridge, Cambridge University Press.

Dorian, Nancy, 1977. " The Problem of the Semi-Speaker in Language Death », Linguistics 15 (191), 23-32.

- 1981. "Language Death: The Life Cycle of a Scottish Gaelic Dialect », Language 58 (2), 432-435.

- 1982. « Defining the Speech Community to Include its Working Margins », Romaine, Suzanne (éd.), Sociolinguistic Variation in Speech Communities, London, Edward Arnold, 25-33.

- 1986. «Abrupt Transmission Failure in Obsolescing Language: How Sudden the Tip to the Dominant Language in Communities and Families? », Nikiforidu, V. M., Van Clay, M., Niepokuj, M. \& Feder, D. (éd.), Proceedings of the 12th Annual Meeting of the Berkeley Linguistics Society, Berkeley, Berkeley Linguistics.

Dressler, Wolfgang, 1981. "Language shift and language death- A protean challenge for the linguist », Folia Linguistica 15 (1-2), 5-28.

Elmendorf, William, 1981. « Last Speakers and Language Change: Two Californian Cases », Anthropological Linguistics 23 (1), 36-49.

Evans, Nicholas, 1995. A Grammar of Kayardild: With Historical-comparative Notes on Tangkic, Berlin, Mouton de Gruyter.

— 2001. « The last speaker is dead? Long live the last speaker! », Newman, P. \& Ratliff, M. (éd.), Linguistic Fieldwork, Cambridge, UK, Cambridge University Press, 250-281.

Fishman, Joshua Aaron, 1991. Reversing Language Shift: Theoretical and Empirical Foundations of Assistance to Threatened Languages, Clevelond, Multilingual Matters.

García de León, Antonio, 1971. «El ayapaneco: una variante del zoqueano en la chontalpa tabasqueña », Anales del Museo Nacional de México 2, 209-224.

Guillaume, Antoine, 2010. « Documentation du reyesano de Bolivie: portraits des derniers locuteurs », Faits de Langues 35/36, 266-286.

Hagège, Claude, 2000. Halte à la mort des langues, Paris, Odile Jacob.

Hale, Ken, Krauss, Michael, Watahomigie, Lucille, Yamamoto, Akira, Craig, Colette, Masayesva, Jeanne, \& England, Nora, 1992. « Endangered Languages », Language 68 (1), $1-42$.

Harvey, Mark, 1993. «A fictitious wife », Hodge, Dino (éd.), Did you Meet any Malagas? A homosexual History of Australia's Tropical Capital, Darwin, Little Gem Publications, 153-164.

Hill, Jane, \& Hill, Kenneth C., 1977. « Language death and relexification in Tlaxcalan Nahuatl », International Journal of the Sociology of Language 12, 55-70. 
Himmelmann, Nikolaous P., 1998. «Documentary and descriptive linguistics », Linguistics, 36(1), 161-195.

- 2004. «Documentary and descriptive linguistics », Sakiyama, Osamu \& Fubito, Endo (éd.), Lectures on Endangered Languages 5: From Tokyo and Kyoto Conference 2002, ELPR 2004 C005, Kyoto, ELPR, 37-83.

Hymes, Dell, H., 1972. « On communicative competence », Pride, J.B \& Holmes, J. (ed.), Sociolinguistics. Selected readings, Harmondsworth, Penguin, 269-293.

Lewis, Paul M., \& Simons, Gary F., 2010. «Assessing endangerment: Expanding Fishman's GIDS », Revue Roumaine de Linguistique 55 (2), 103-120.

Miller, W., 1971. " The death of language, or Serendipity among the Shoshoni ", Anthropological Linguistics, 13, 114-120.

O'Rourke, Bernadette, \& Pujolar, Joan, 2015. « New speakers and processes of new speakerness across time and space », Applied Linguistics Review, 6 (2), 145-150.

O'Rourke, Bernadette, Pujolar, Joan, \& Ramallo, Fernando 2014. " New speakers of minority languages: the challenging opportunity ", International Journal of the Sociology of Language 231, 1-20.

Palancar, Enrique, 2009. Gramática y textos del hñöñhö: otomí de San Ildefonso Tultepec, Querétaro, México, D.F, Plaza y Valdés.

Quesada, J. Diego, 2000. « Synopsis of a Boruca terminal speaker », Amerindia 25, 65-86.

Radin, Paul, 1929. A Grammar of the Wappo Language, University of California Press.

Rangel, Jhonnatan, en cours. « Variations linguistiques et langue menacée de disparition. Le cas du numte ote ou ayapaneco dans l'état de Tabasco, Mexique », Thèse de doctorat en sciences du langage, sous la direction de Claudine Chamoreau, Paris, Institut National des Langues et Civilisations Orientales.

Reber, Lu \& Webb, Lilian, 1961. « Unpublished field notes », Mexico, Summer Institute of Linguistics Mexico, 1-2

Sasse, Hans-Jürgen, \& Brenzinger, Matthias, 1992. " Theory of language death and Language decay and contact-induced change: similarities and differences », Brenzinger, Matthias (ed.), Language death: Factual and Theoretical Explorations with Special Reference to East Africa, Berlin, Mouton de Gruyter, 59-80.

Sawyer, Jeese O., 1965. English-Wappo vocabulary, Berkeley, University of California Press.

Suslak, Daniel F., 2011. «Ayapan Echoes: Linguistic Persistence and Loss in Tabasco, Mexico », American Anthropologist 113, 569-581.

Tsunoda, Tasaku, 2006. Language endangerment and language revitalization: an introduction, Berlin, Mouton de Gruyter.

UNESCO ad Hoc Expert Group on Endangered Languages. 2003. Language vitality and endangerment [en ligne]. Disponible sur : http://www.unesco.org/new/fr/culture/themes/endangered-languages/language-vitality/ [Consulté le 17 mars 2016].

Wichmann, Søren, 1995. The relationship among the Mixe-Zoquean languages of Mexico, Salt Lake City, University of Utah Press.

Woodbury, Anthony C., 2003. "Defining Documentary Linguistics », Ausin, Peter (ed.), Language Documentation and Description, London, SOAS The Hans Rausing Endangered Languages Project, 35-51.

Zavala, Roberto, 2000. Inversion and other topics in the grammar of Olutec (Mixean), Thèse de doctorat, University of Oregon.

- 2015. «Instrumentos y comitativos en las lenguas de la familia mixe zoque », Amerindia 37 (2), 189-228. 